\title{
Removal of giant intraosseous meningioma followed by cranioplasty using a custom-made bioceramic implant: case report
}

\author{
Jimmy Sundblom, MD, PhD, ${ }^{1}$ Daniel Nowinski, MD, PhD, ${ }^{2}$ Olivera Casar-Borota, MD, $\mathrm{PhD},{ }^{3,4}$ and \\ Mats Ryttlefors, MD, PhD'
}

${ }^{1}$ Department of Neuroscience, Neurosurgery, Uppsala University Hospital; ${ }^{2}$ Department of Surgical Sciences, Plastic Surgery, Uppsala University Hospital; ${ }^{3}$ Department of Immunology, Genetics and Pathology, Uppsala University; and ${ }^{4}$ Department of Clinical Pathology, Uppsala University Hospital, Uppsala, Sweden

\begin{abstract}
Intraosseous meningioma of the chordoid type is a rare clinical entity. Radical surgical removal and subsequent cranioplasty is the treatment of choice. Here, the authors report a severe case involving more than $70 \%$ of the calvarial surface area, which was removed and repaired using a prefabricated custom-made, titanium-reinforced, bioceramic implant and bone-cutting guides. Tumor removal and good esthetic outcome were achieved, along with a $17.1 \%$ increase of intracranial volume. Bioceramic implants have shown promising initial results and may represent an important new tool in the surgeon's armamentarium.
\end{abstract}

https://thejns.org/doi/abs/10.3171/2018.4.JNS1850

KEYWORDS intraosseous; meningioma; cranioplasty; bioceramic implant; oncology

$\mathrm{E}$ XTRADURAL meningiomas comprise approximately $2 \%$ of all meningiomas, and of these approximately two-thirds are primary intraosseous meningiomas, ${ }^{5}$ which may involve underlying dura mater and extracranial soft tissue. ${ }^{18}$ The knowledge of this entity is based on case reports or case series describing radiological and histopathological features. ${ }^{10}$ The optimal treatment is no different from treatment of other types of meningioma-total resection including the affected bone, dura, and other tissue while preserving neural tissue integrity ${ }^{13}$ Intraosseous meningioma with a chordoid growth pattern (WHO grade II) is exceedingly rare, with only 2 cases described in the literature. One-year follow-up after surgical management of the patients reported showed no signs of tumor recurrence. , $^{8,17}$

With extensive bone involvement, complete bone resection and reconstruction is necessary. When repairing the subsequent very large skull defects, custom-made implants are an attractive option. Bone-cutting guides facilitate removal of bone for precise fitting of the implant. ${ }^{2,3}$ In a near total cranioplasty the risk of infection must be minimized due to the risks associated with removal of the implant.

Custom-made implants with inert materials such as methyl methacrylate, porous polyethylene (Medpor), polyetheretherketone, or titanium are widely used. These materials offer necessary structural strength and are nontoxic. They elicit a foreign body reaction, ${ }^{1}$ and are sensitive to bacterial contamination during surgery. Accordingly, the most common complication of cranioplasty with nonautologous bone is infection, reported in ranges from $10 \%$ to $30 \% .9,12,16$

Due to the high failure rates of traditional implants there is an increased interest in new materials for cranioplasty. Recent developments include fiberglass-reinforced composite materials and bioceramic compounds mimicking the nonorganic portion of the bony matrix, with osteoconductive and/or osteoinductive properties., ${ }^{711,15}$ Bioceramics have shown lower risk for infection, but early products have been lacking in the structural strength necessary to cover large-sized defects, and generally complete ossification could not be expected to occur. Recently, a new implant has been developed combining the properties of a bioceramic component with the structural strength of titanium. A new mosaic tile design has been suggested to increase osteoconductivity further. The incorporated titanium mesh also allows for custom-made designs with complex 3D shapes. ${ }^{6,7}$

We report a case of extremely large intraosseous meningioma involving more than $70 \%$ of the cranial convexity. This lesion was resected in a 2-stage procedure aided by predesigned bone-cutting guides and concluded with primary cranioplasty performed using a mosaic tile-design, titanium-reinforced bioceramic implant. 


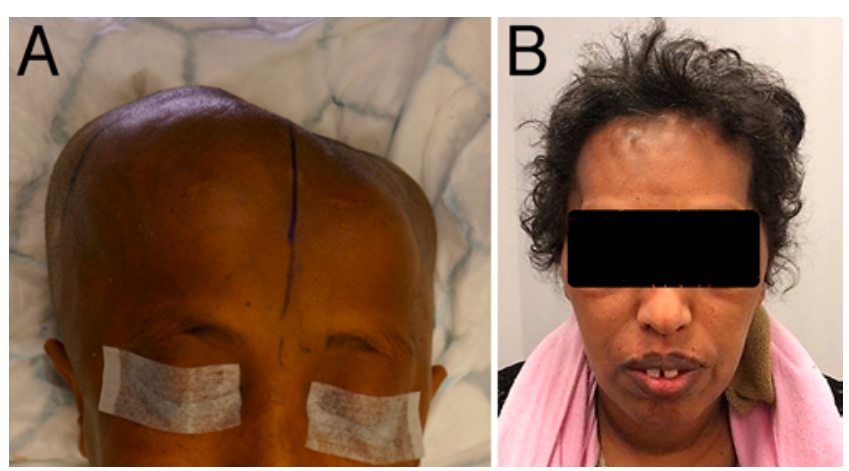

FIG. 1. A: Preoperative image showing severe bulging of frontal bone and the whole of the right calvaria. B: 6 months postoperatively, a good cosmetic outcome is seen. Note the slight outline of the cranioplasty segments, probably due to the necessary removal of periosteum. In our experience, this outline will disappear during the 1st year after surgery. Figure is available in color online only.

\section{Case Report}

History and Examination

A 39-year-old woman presented to the department with tenderness of the scalp and severe headache. She was a recent migrant from East Africa, and had a 5-year history of swelling in the right temporal bone, which had subsequently progressed significantly. Traditional medicinal treatments including chipping of bone had been administered.

A severe expansion of almost all calvarial bones on the right side was seen, as well as involvement of frontal and parietal bones on the left side (Fig. 1A). Results of neurological examination were normal except for bilateral anosmia. The scalp was not adherent to underlying bone. Ophthalmological examination showed bilateral papilledema with preserved vision. Visual field analysis (Humphrey) revealed concentrically diminished visual fields bilaterally that were pronounced on the right side, with a visual field index of $41 \%$.

CT scanning revealed hyperostosis and probable hyperostotic intraosseous meningioma involving the frontal, parietal, and temporal bones on both sides, with most extensive involvement of the right side. The upper orbital rim and frontal sinus were involved. Also, small intracranial meningiomas of the lateral left orbital roof and confluence of sinuses were visible.

MRI revealed extensive dural involvement with thickened dura and contrast enhancement underneath all affected bone. The intraosseous component on the right side showed a spiculated appearance, suggestive of grade II meningioma. ${ }^{10}$ Invasion of extracranial soft tissues adjacent to the spiculated parts of the tumor was suspected. The sagittal sinus was patent (Fig. 2).

Due to the very extensive involvement, a procedure with primary tumor removal and secondary cranioplasty was deemed unsuitable due to the risks associated with such a large defect. Reconstruction of such an extensive defect with methyl methacrylate or prefabricated noncustomized implants would not produce a cosmetically satisfying result, and would carry high risks for implantrelated complications.
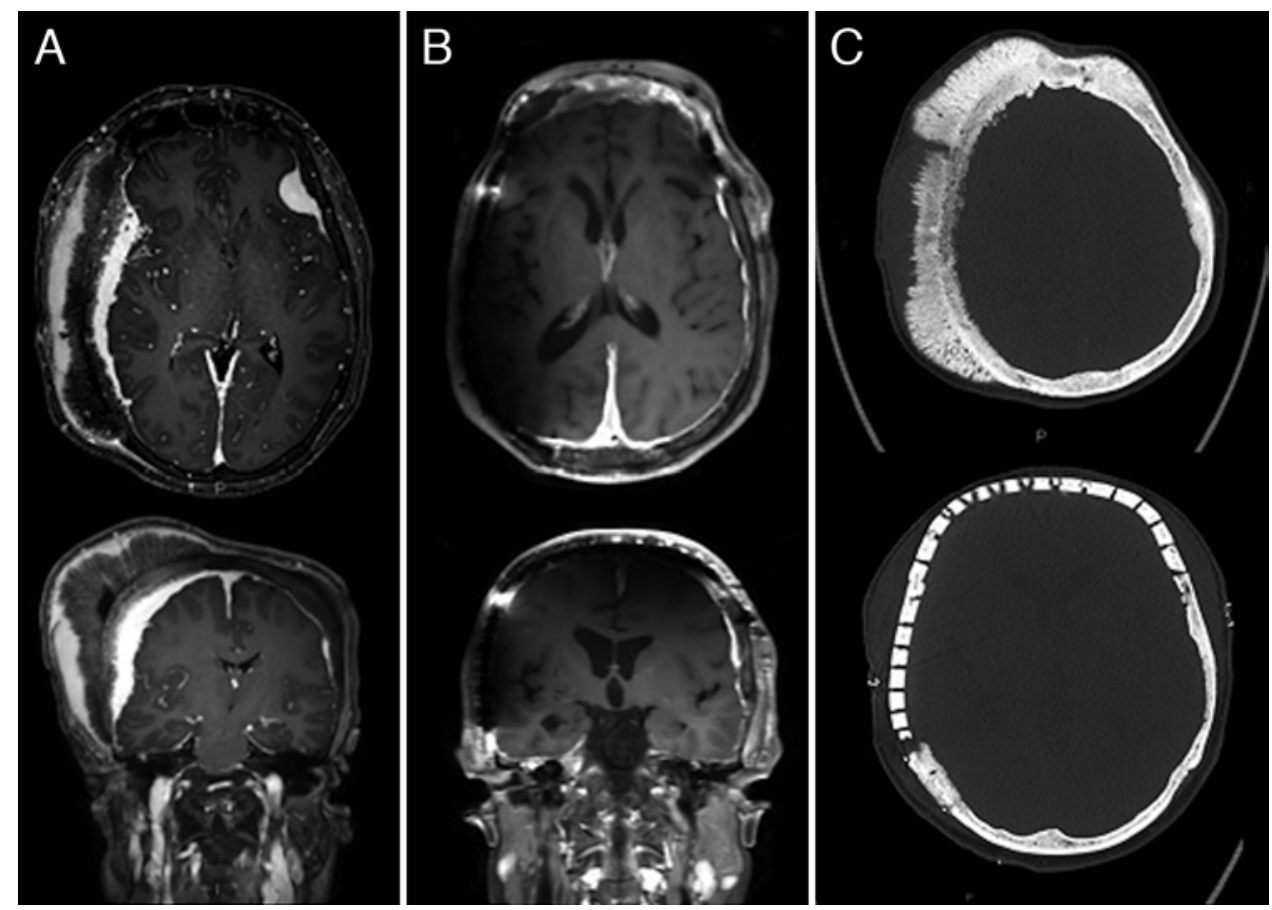

FIG. 2. A: Preoperative MRI showing extensive spiculated involvement of bone, dural enhancement, and soft-tissue invasion on the right side. Also, note smaller intradural meningiomas of the lateral sphenoid wing and confluence of sinuses. B: Postoperative MRI showing removal of tumor and increase of intracranial volume and ventricle size. Contralateral meningioma was unchanged. C: Preoperative CT (upper) showing extension of bone involvement. Postoperative CT (lower) showing cranioplasty in place. 
A
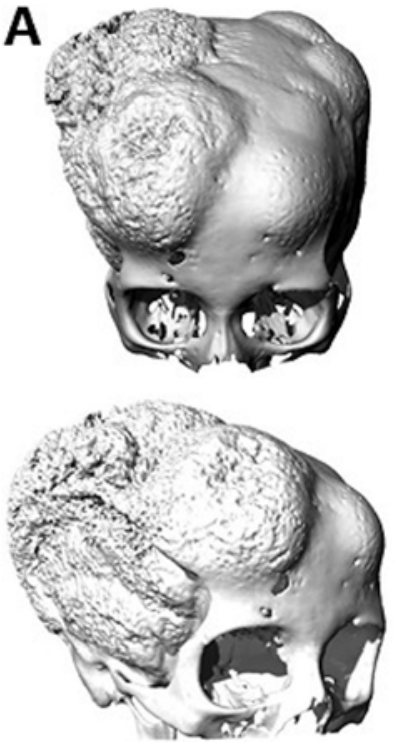

B
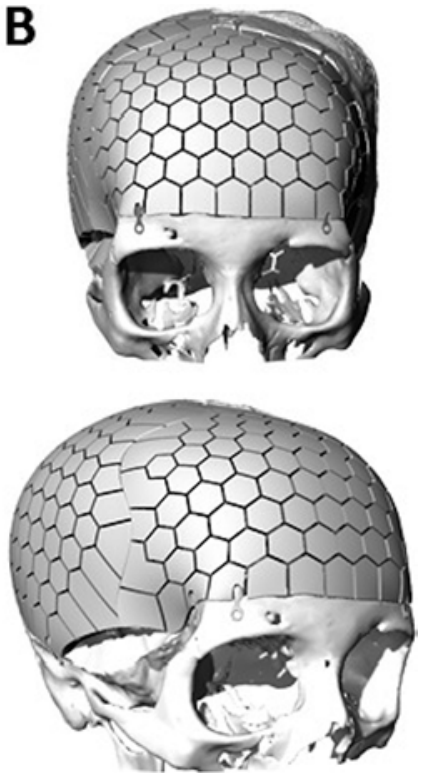

FIG. 3. A: 3D renderings of the patient's skull at presentation. B: Planned cranioplasty design.

Extensive bifrontal and right-sided tumor resection via a combined approach, with primary cranioplasty performed using a bioceramic implant (OssDsign), was the chosen strategy. The smaller, isolated involvement of the left side was left untreated initially, but with care taken during design of implants and incision to facilitate removal at a later stage. The implant was designed in two separate pieces; one covering the frontal region bilaterally and the other covering the temporoparietal region on the right side (Fig.
3). Titanium bone-cutting guides were designed to aid the resection. Preoperative embolization was considered but not deemed suitable, due to the risk of compromising the blood circulation to adjacent bone and scalp.

\section{Operation}

After induction of anesthesia, the scalp was shaved and head fixed in a vacuum pillow to allow for positional adjustment during surgery. A bicoronal incision was made and subgaleal dissection was performed. Periosteum on the right side was affected by the tumor and was removed (Fig. 4A). Inspection of extracranial soft tissue was performed and affected tissue, including periosteum and parts of the temporal muscle, was removed. Hyperostotic bone was removed with a high-speed drill (Midas Rex Acorn; Medtronic) until the bone-cutting guide could be fitted. Burr holes were placed straddling the sagittal sinus and anteriorly and posteriorly to the sphenoid wing bilaterally. Underlying dura was dissected free and bifrontal craniectomy was performed. After bone removal, the patient was hemodynamically unstable despite meticulous continuous management of bleeding. After volume and blood replacement the procedure could have continued, but a decision was made to stage the tumor resection.

After the bone flap was removed, cranialization of the frontal sinuses was performed using curettes and a high-speed drill. After removal of all mucosa, sinus was plugged with autologous bone from the iliac crest, SeptoColl (Zimmer Biomet), fibrin glue (Tisseel; Baxter), and fat obtained from the bone donor site incision (Fig. 4B and C). Concomitantly, microsurgical removal of the intradural meningioma on the left orbital roof was performed, including dural resection with a 5-mm margin. Bifrontal
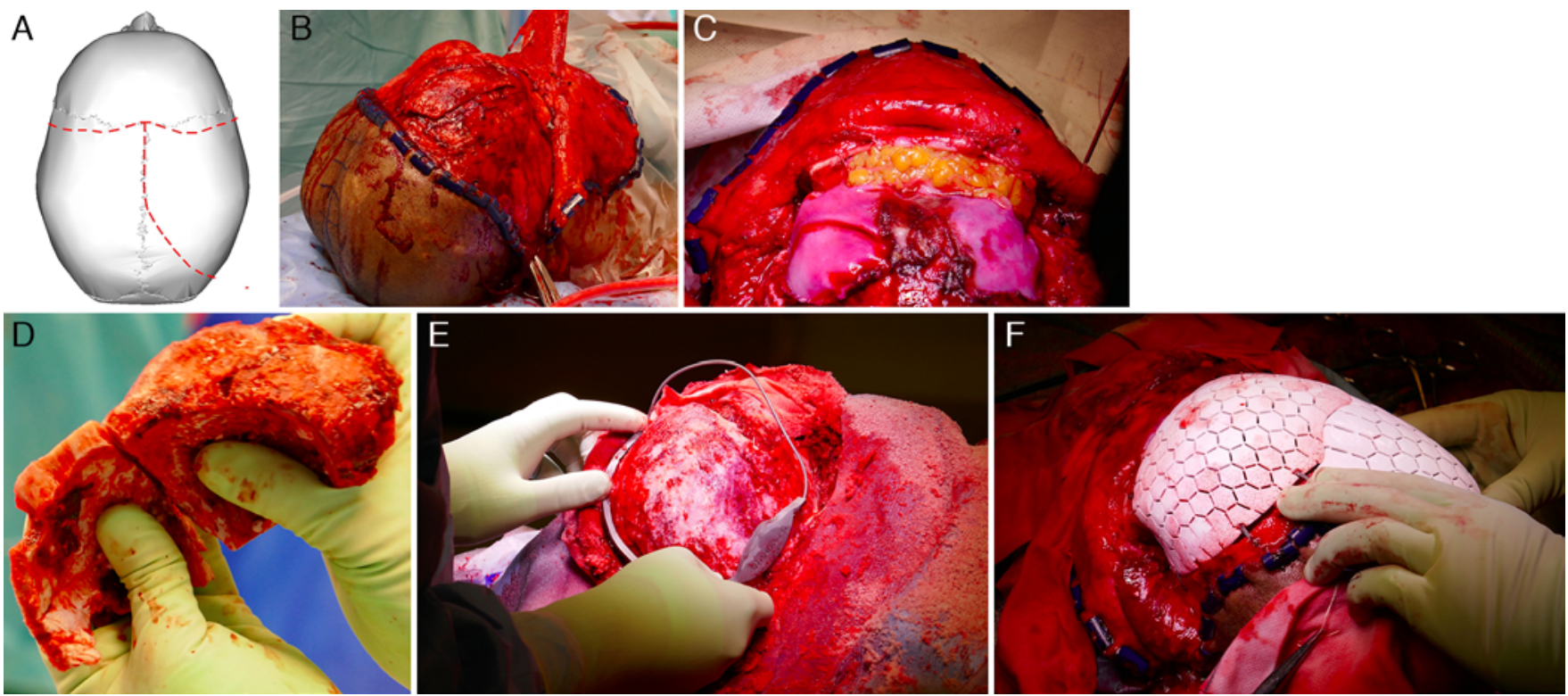

FIG. 4. A: Planned incision lines drawn on a model skull. B: Intraoperative photograph showing bicoronal incision. Intact periosteum on the left side was used to cover frontal sinus. C: After removal of frontal bone bilaterally, fat tissue packing of frontal sinus is seen as well as temporary bilateral duraplasty with DuRepair. D: Removed frontal part of intraosseous meningioma. E: A bonecutting guide was used to trim edges of bone for the intended cranioplasty. F: Primary cranioplasty with custom-made bioceramic implants. Figure is available in color online only. 


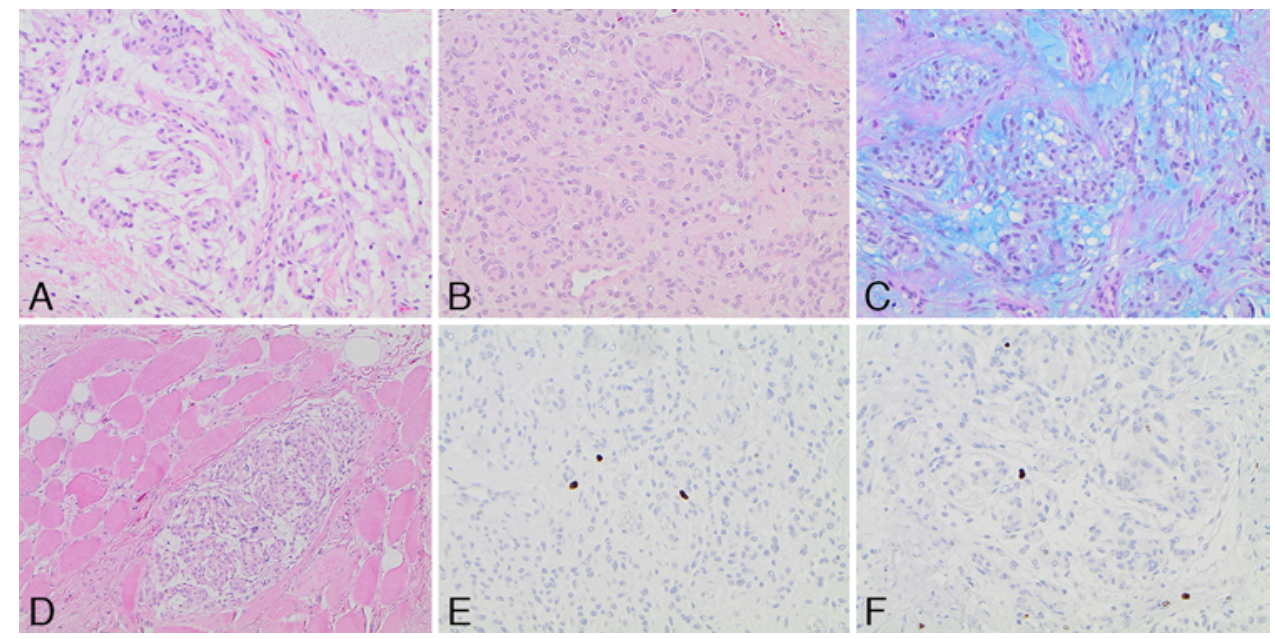

FIG. 5. Photomicrographs showing histopathological findings. A: Predominant chordoid pattern in the tumor specimen from the secondary surgery. H \& E. B: In contrast, meningioma cells without atypia from right-sided supraorbital intracranial meningioma. H \& E. C: Vacuolated tumor cells in the mucin-rich extracellular matrix characteristic of chordoid meningioma. Alcian blue-periodic acid-Schiff staining. D: Extracranial muscle tissue infiltrated by the tumor. H \& E. E: MIB-1 staining from the first surgery (Ki-67). F: MIB-1 staining from the second surgery (Ki-67). Original magnification $\times 200$ in all panels. Figure is available in color online only.

duraplasty was done with DuRepair (Medtronic), followed by skin closure. The patient recovered fully, and the second stage was performed 13 days later.

The bifrontal incision was opened, and a midline incision posteriorly to the vertex, then curving caudally on the right side, was added. Skin flaps were lifted and periosteum removed (Fig. 4D). Thinning of hyperostotic bone was performed with a high-speed drill, followed by piecemeal removal of bone with rongeurs. Meticulous hemostasis of bone and underlying dura was performed. The thickened and affected dura was removed microsurgically, while a large free fascia lata flap was lifted from the right thigh. The flap was implanted with running 4-0 Vicryl suture in a watertight fashion. The bone-cutting guides were fitted (Fig. 4E) and further bone removal around the edges was performed using a high-speed drill. The bioceramic implant was fixated with titanium screws (Fig. 4F). We decided against removal of expanded skin to easily achieve tension-free closure.

\section{Pathological Findings}

Microscopic examination of the specimen from the first surgery confirmed extensive growth of the meningioma in the resected cranial bone. Histopathological appearance of the intradural tumor components corresponded to meningothelial meningioma, with only focal presence of chordoid differentiation. The chordoid pattern was predominant in the specimen from the second surgery (Fig. $5 \mathrm{~A}$ and $\mathrm{B}$ ), leading to the classification of the tumor as a chordoid meningioma, WHO grade II, despite the absence of mitoses and low proliferation index (Ki-67 1\%-2\%). In addition, a massive infiltration of the meningioma into the extracranial soft tissues was demonstrated (Fig. 5C).

\section{Postoperative Course}

Postoperatively, the patient was hemiparetic in the left side, and MRI was performed demonstrating a small infarction in the putamen on the right side. MRI also showed complete removal of the tumor components addressed. The patient recovered quickly, and was fully ambulatory, with normal results on a neurological examination performed 14 days postoperatively. Due to a subgaleal fluid collection, a headdress was used for 1 month postoperatively. The fluid collection cleared, and at follow-up 6 weeks postoperatively wound healing had been uneventful and a good cosmetic outcome had been achieved. Ophthalmological follow-up showed improvement of papilledema, with an increase in visual field index on the right side from $41 \%$ to $96 \%$. Calculated from pre- and postoperative $\mathrm{CT}$ scans, the intracranial volume was increased by $23.1 \mathrm{~cm}^{3}$, an increase in the intracranial volume of $17.1 \%$.

MRI studies obtained 5 months after surgery showed no signs of tumor recurrence at the site of surgery and no signs of infection. The patient was well and without clinical signs of infection at follow-up 6 months postoperatively (Fig. 1B).

\section{Discussion}

Dealing with large tumors of the calvaria is challenging from many perspectives. This case, showing extreme progression due to lack of medical resources in the patient's native country, illustrates these challenges. We decided on an approach initially targeting the frontal and right-sided involvement, achieving total removal of this component. We also radically removed a small asymptomatic intradural meningioma to avoid having to remove the frontal cranioplasty at a later stage. This plan was supported by the MRI appearance of the intraosseous components, of which only the right-sided tumor showed a spiculated appearance suggestive of higher-grade meningioma. ${ }^{10}$ During the design stage, a bony rim in the midline posterior to the bregma was preserved to allow for later removal of the left-sided intraosseous meningioma and fitting of cranioplasty, also providing structural integrity and support for the superior sagittal sinus. 
To achieve total resection of the frontal bone involvement, the frontonasal sinuses had to be entered, necessitating a thorough obliteration of the sinuses to decrease the risk of postoperative infection and implant failure. Implant failure with subsequent removal of the cranioplasty would put the patient at great risk due to the size of the defect.

Because early reports of bioceramic implants with bone-growing properties have been promising, this was our initial choice. An explant rate of $2.4 \%$ was recently reported in a cohort of 41 patients (Engstrand T, Birgersson U, Kihlström L: Clinical outcome analysis of 41 consecutive patients treated with bone regenerative calcium-phosphate based cranial implants, presented at the Congress of Neurological Surgeons, 2017 Annual Meeting, Boston, Massachusetts, October 7-11, 2017). Several cases with infection or exposure of the custom-made implant have also been successfully managed with systemic antibiotic therapy and adjuvant surgery without removal of the flap (Mielke D, Engstrand T, Birgersson U, et al: Does wound dehiscence in cranioplasty with customized implants always warrant explantation?, presented at the Deutsche Gesellschaft für Neurochirurgie-Society of British Neurological Surgeons Joint Meeting, Magdeburg, Germany, May 14-17, 2017), which, if infection was suspected, would be a great advantage in our case.

After surgery, pathological investigation confirmed involvement of the extracranial soft tissues but not of the skin. This had been considered preoperatively and a plan had been made for free flap placement in the event of skin involvement. The removal of such a large tumor proved a time-consuming endeavor with severe blood loss, and the decision to stage the procedure was proven correct.

The chordoid meningioma subtype is considered a WHO grade II meningioma. ${ }^{4,8}$ This necessitates careful follow-up and will also affect treatment choices for the remaining tumor components. In the case of local tumor recurrence warranting treatment, surgery will be the initial choice. Radiotherapy is indicated for grade II meningiomas ${ }^{14}$ but we opted for a wait-and-scan approach because additional tumor components may warrant surgical treatment in a relatively short period of time. This case illustrates the possibility of multidisciplinary microsurgical treatment of giant skull tumors with good functional and cosmetic outcome. Bioceramic implants are a promising new development, increasing the surgical options available when managing these patients.

\section{References}

1. Brett-Major DM, Baraniak SM, Gilhooly JE, Christensen RL, Grant GT, Armonda RA, et al: Foreign-body reaction mimicking postneurosurgical infection after cranioplasty. Mil Med 173:697-699, 2008

2. Broeckx CE, Maal TJJ, Vreeken RD, Bos RRM, Ter Laan M: Single-step resection of an intraosseous meningioma and cranial reconstruction: technical note. World Neurosurg 108:225-229, 2017

3. Carolus A, Weihe S, Schmieder K, Brenke C: One-step $\mathrm{CAD} / \mathrm{CAM}$ titanium cranioplasty after drilling templateassisted resection of intraosseous skull base meningioma: technical note. Acta Neurochir (Wien) 159:447-452, 2017

4. Choy W, Ampie L, Lamano JB, Kesavabhotla K, Mao Q, Parsa AT, et al: Predictors of recurrence in the management of chordoid meningioma. J Neurooncol 126:107-116, 2016
5. Elder JB, Atkinson R, Zee CS, Chen TC: Primary intraosseous meningioma. Neurosurg Focus 23(4):E13, 2007

6. Engstrand T, Kihlström L, Lundgren K, Trobos M, Engqvist $\mathrm{H}$, Thomsen P: Bioceramic implant induces bone healing of cranial defects. Plast Reconstr Surg Glob Open 3:e491, 2015

7. Engstrand T, Kihlström L, Neovius E, Skogh AC, Lundgren TK, Jacobsson H, et al: Development of a bioactive implant for repair and potential healing of cranial defects. J Neurosurg 120:273-277, 2014

8. Hayashi T, Haba R, Kushida Y, Kadota K, Katsuki N, Bando $\mathrm{K}$, et al: Cytopathologic features of orbital intraosseous chordoid meningioma: report of a case and distinction from other myxoid/mucoid tumors. Diagn Cytopathol 38:818-821, 2010

9. Honeybul S, Ho KM: Cranioplasty: morbidity and failure. Br J Neurosurg 30:523-528, 2016

10. Ilica AT, Mossa-Basha M, Zan E, Vikani A, Pillai JJ, Gujar $S$, et al: Cranial intraosseous meningioma: spectrum of neuroimaging findings with respect to histopathological grades in 65 patients. Clin Imaging 38:599-604, 2014

11. Posti JP, Piitulainen JM, Hupa L, Fagerlund S, Frantzén J, Aitasalo KMJ, et al: A glass fiber-reinforced composite-bioactive glass cranioplasty implant: a case study of an early development stage implant removed due to a late infection. J Mech Behav Biomed Mater 55:191-200, 2015

12. Punchak M, Chung LK, Lagman C, Bui TT, Lazareff J, Rezzadeh K, et al: Outcomes following polyetheretherketone (PEEK) cranioplasty: systematic review and meta-analysis. J Clin Neurosci 41:30-35, 2017

13. Rogers L, Barani I, Chamberlain M, Kaley TJ, McDermott M, Raizer J, et al: Meningiomas: knowledge base, treatment outcomes, and uncertainties. A RANO review. J Neurosurg 122:4-23, 2015

14. Rogers L, Zhang P, Vogelbaum MA, Perry A, Ashby LS, Modi JM, et al: Intermediate-risk meningioma: initial outcomes from NRG Oncology RTOG 0539. J Neurosurg [epub ahead of print October 6, 2017. DOI: 10.3171/2016.11.JNS161170]

15. Staffa G, Nataloni A, Compagnone C, Servadei F: Custom made cranioplasty prostheses in porous hydroxy-apatite using 3D design techniques: 7 years experience in 25 patients. Acta Neurochir (Wien) 149:161-170, 2007

16. Thien A, King NK, Ang BT, Wang E, Ng I: Comparison of polyetheretherketone and titanium cranioplasty after decompressive craniectomy. World Neurosurg 83:176-180, 2015

17. Tokgoz N, Oner YA, Kaymaz M, Ucar M, Yilmaz G, Tali TE: Primary intraosseous meningioma: CT and MRI appearance. AJNR Am J Neuroradiol 26:2053-2056, 2005

18. Yun JH, Lee SK: Primary osteolytic intraosseous atypical meningioma with soft tissue and dural invasion: report of a case and review of literatures. J Korean Neurosurg Soc 56:509-512, 2014

\section{Disclosures}

Dr. Ryttlefors received a lecture honorarium from OssDsign during 2016.

\section{Author Contributions}

Conception and design: Sundblom, Ryttlefors. Acquisition of data: all authors. Analysis and interpretation of data: all authors. Drafting the article: Sundblom. Critically revising the article: Nowinski, Casar-Borota, Ryttlefors. Reviewed submitted version of manuscript: Nowinski, Casar-Borota, Ryttlefors. Approved the final version of the manuscript on behalf of all authors: Sundblom. Study supervision: Ryttlefors.

\section{Correspondence}

Jimmy Sundblom: Uppsala University Hospital, Uppsala, Sweden. jimmy.sundblom@neuro.uu.se. 